\title{
Metabolic engineering of Rhodopseudomonas palustris for the obligate reduction of $n$-butyrate to $n$-butanol
}

\author{
Devin F. R. Doud', Eric C. Holmes ${ }^{1}$, Hanno Richter ${ }^{1}$, Bastian Molitor ${ }^{1}$, Georg Jander ${ }^{2,3}$ \\ and Largus T. Angenent ${ }^{1,3,4^{*}}$
}

\begin{abstract}
Background: Rhodopseudomonas palustris is a versatile microbe that encounters an innate redox imbalance while growing photoheterotrophically with reduced substrates. The resulting excess in reducing equivalents, together with ATP from photosynthesis, could be utilized to drive a wide range of bioconversions. The objective of this study was to genetically modify $R$. palustris to provide a pathway to reduce $n$-butyrate into $n$-butanol for maintaining redox balance.

Results: Here, we constructed and expressed a plasmid-based pathway for $n$-butanol production from Clostridium acetobutylicum ATCC 824 in R. palustris. We maintained the environmental conditions in such a way that this pathway functioned as the obligate route to re-oxidize excess reducing equivalents, resulting in an innate selection pressure. The engineered strain of $R$. palustris grew under otherwise restrictive redox conditions and achieved concentrations of $1.5 \mathrm{mM} n$-butanol at a production rate of $0.03 \mathrm{~g} \mathrm{~L}^{-1}$ day $^{-1}$ and a selectivity (i.e., products compared to the consumed substrate) of close to $40 \%$. Since the theoretical maximum selectivity is $45 \%$, the engineered strain converted close to its maximum selectivity.
\end{abstract}

Conclusions: The innate redox imbalance of $R$. palustris can be used to drive the reduction of $n$-butyrate into n-butanol after expression of a plasmid-based enzyme from a butanol-producing Clostridium strain.

Keywords: Metabolic engineering, Redox-driven obligate reduction, $n$-Butanol, Rhodopseudomonas palustris

\section{Background}

Rhodopseudomonas palustris, which is a member of the purple non-sulfur bacteria group, is often noted for its metabolic versatility. One of these metabolic activities, which is $\mathrm{H}_{2}$ production by photofermentation, utilizes organic carbon under anaerobic conditions with light and has become an attractive application for bioenergy production [1]. The ability to produce $\mathrm{H}_{2}$ at high concentrations is driven by an excess of reducing equivalents that

\footnotetext{
*Correspondence: l.angenent@uni-tuebingen.de

${ }^{4}$ Center for Applied Geosciences, University of Tübingen, Hölderlinstr. 12, 72074 Tübingen, Germany
}

Full list of author information is available at the end of the article accumulate during photoheterotrophic growth [2]. Here, we aim to exploit the excess reducing equivalents generated during photoheterotrophic growth to drive an engineered reduction reaction for the obligate production of the liquid fuel $n$-butanol (hereafter butanol).

When growing on acetate, McKinlay and Harwood [1] reported that approximately half of all reducing equivalents that were generated were used for biosynthesis, while the other half required re-oxidation by some alternate pathway such as $\mathrm{CO}_{2}$ fixation, $\mathrm{H}_{2}$ evolution, or a combination of both. These two pathways were found to be complementary in this role, and when one route was made unavailable, the other facilitated the re-oxidation 
of all reducing equivalents with minimal changes occurring elsewhere in the central metabolism. However, when fed a more reduced substrate, such as $n$-butyrate (hereafter butyrate), it was observed that under non- $\mathrm{H}_{2}$ evolving conditions, $R$. palustris could only grow if exogenous $\mathrm{CO}_{2}$ was supplemented into the medium $[2,3]$. This finding demonstrates that under these conditions, $R$. palustris generates more reducing equivalents than available electron sinks can accommodate, and ultimately suffers a lethal redox imbalance. Numerous studies have used these principles to improve $\mathrm{H}_{2}$ yields from photofermentation using R. palustris [4-6].

Recently, Fixen et al. [7] engineered a strain of $R$. palustris to utilize excess reducing equivalents to reduce $\mathrm{CO}_{2}$ into $\mathrm{CH}_{4}$ by remodeling the nitrogenase enzyme active site and expressing the modified gene from the chromosome. A NifA* strain of $R$. palustris was chosen as the host because it expresses the nitrogenase gene constitutively even in the presence of $\mathrm{NH}_{3}$. The result was a light-dependent $R$. palustris strain that produced $\mathrm{CH}_{4}$ with ATP coming from cyclic photophosphorylation and electrons coming from either an organic substrate or thiosulfate. This reduction is, however, not obligatory and a large excess of $\mathrm{H}_{2}$ was produced by the nitrogenase compared to $\mathrm{CH}_{4}$ [7]. Another attractive route to redirect the excess reducing equivalents and to maintain redox balance when growing on butyrate is to produce the fuel butanol through an obligate reduction reaction. The advantage of the obligate reduction is the lack of side products to achieve a high selectivity (i.e., products compared to the consumed substrate).

Butanol is an attractive biofuel molecule due to its higher energy density, lower volatility, and complete intersolubility with traditional fuels compared to ethanol [8]. Butanol is traditionally produced via fermentation of monosaccharides by certain strains of Clostridia. Clostridium acetobutylicum ATCC 824, which has been widely studied for this activity, is generally seen as the model organism for solvent production and previously has been used as a source of genes for engineered butanol production [9-11]. In C. acetobutylicum, reduction of butyrate proceeds through a two-phase pathway. First, butyrate is activated to butyryl-CoA, which can occur by two different routes: (1) a two-step butyrate kinase/phosphotransbutyrylase reaction (Fig. 1); or (2) a one-step reaction with butyrate/acetoacetyl-CoA transferase transferring a CoA group from acetoacetyl-CoA to butyrate. Second, butyryl-CoA is reduced to butanol through the butyraldehyde intermediate $[12,13]$. The AdhE2 enzyme from C. acetobutylicum ATCC 824 (hereafter AdhE2 $2_{824}$ and $a d h E 2_{824}$ for its gene) reduces butyrylCoA to butyraldehyde and subsequently butanol (Fig. 1) [14]. Analysis of AdhE2 $2_{824}$ has revealed that the protein is

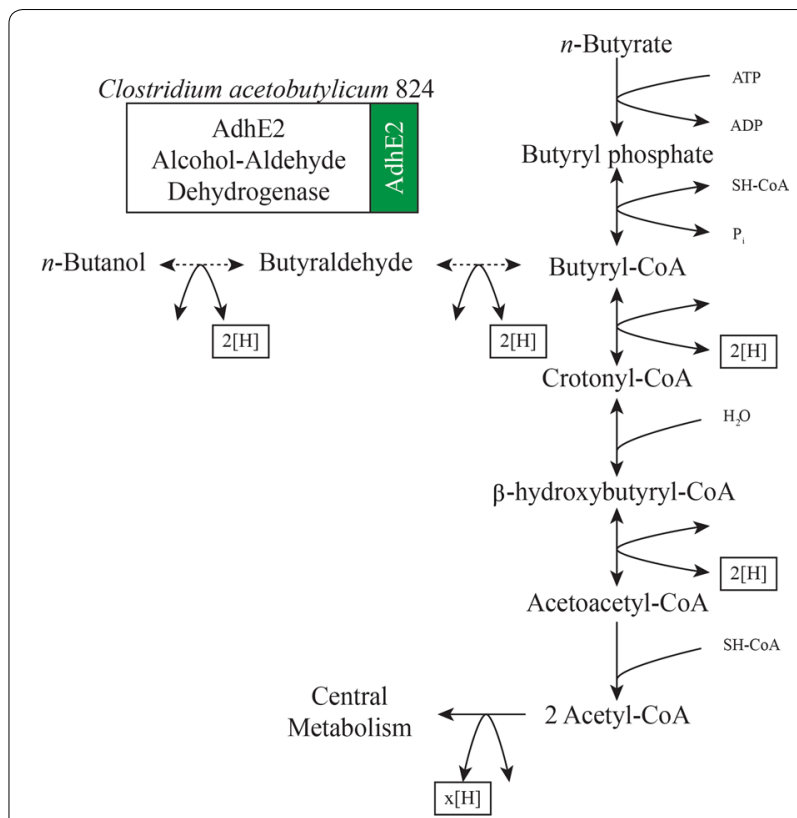

Fig. 1 Butyrate metabolism and the proposed route for butanol production in R. palustris by the activity of the butyryl-CoA to butanol AdhE2 enzyme. Dotted arrows represent the proposed pathway and solid arrows indicate native metabolism

a bifunctional NADH-dependent fusion protein with the $\mathrm{N}$-terminal domain retaining highly conserved sequences of the aldehyde dehydrogenase family and the C-terminal domain retaining conserved sequences of the ironcontaining alcohol dehydrogenase family [15]. A second alcohol dehydrogenase enzyme BdhB has also been found to aid in the reduction of butyraldehyde to butanol in C. acetobutylicum, though its activity is not necessary for butanol production [16].

In planning to introduce the butanol production pathway together with the existing catabolism of butyrate by $R$. palustris, we established that before butyrate can be converted to two molecules of acetyl-CoA via anaerobic $\beta$-oxidation, butyryl-CoA is first formed through native butyrate kinase/phosphotransbutyrylase enzymes (Fig. 1). Under photoheterotrophic redox imbalance, the first oxidation reaction for the metabolism of butyrate (i.e., butyrylCoA to crotonyl-CoA in Fig. 1) would likely be inhibited by the thermodynamic limitations of the reduced cofactor pool. Because of this, we anticipated the butyryl-CoA intermediate to accumulate and provide an ideal target for shunting excess reducing equivalents for the production of butanol by introducing only adhE2 224 (the bifunctional aldehyde/alcohol dehydrogenase gene) into $R$. palustris (Fig. 1). After necessary optimizations, the production of butanol indicated that this assumption was correct.

A direct introduction of plasmid-based $a d h E 2_{824}$ did not result in the production of AdhE2 $2_{824}$ (and butanol 
production) due to the major incompatibilities in codon utilization preference between the $C$. acetobutylicum source and the $R$. palustris host. Therefore, we pursued two alternate attempts to introduce the activity of AdhE2 by using: (1) a gene homologous to $a d h E 2_{824}$ from $R$. palustris strain BisB18 (hereafter $a d h E_{\mathrm{BisB} 18}$ and $\mathrm{AdhE}_{\text {BisB18 }}$ for its protein); and (2) a codon-optimized adhE2 $2_{824}$ (hereafter $a d h E 2_{\text {opti }}$ and AdhE2 $2_{\text {opti }}$ for its protein). In all our growth experiments, we omitted $\mathrm{HCO}_{3}{ }^{-}$ $\left(\mathrm{CO}_{2}\right)$ from, and added $\mathrm{NH}_{3}$ to, the growth medium to block the normal routes of shunting reducing equivalents, resulting in an obligate butyrate reduction. This strong selection pressure will only allow growth when butanol production occurs. In our hands, butanol production through the homologous $\mathrm{AdhE}_{\mathrm{BisB18}}$ did not work, but AdhE $2_{\text {opti }}$ enabled recovered growth through butanol production. Subsequently, we were able to increase the butanol selectivity and production rate in further experiments, accomplishing our aim of generating an engineered reduction reaction for the obligate production of the liquid fuel butanol in $R$. palustris.

\section{Methods}

\section{Media and culture conditions}

Rhodopseudomonas palustris CGA009 was cultured in anaerobic butyrate Rhodospirillaceae medium (RM) [17], consisting of $1.64 \mathrm{~g} \mathrm{NaHCO}_{3}, 0.5 \mathrm{~g} \mathrm{KH}_{2} \mathrm{PO}_{4}, 0.5 \mathrm{~g}$ $\mathrm{K}_{2} \mathrm{HPO}_{4}, 0.2 \mathrm{~g} \mathrm{MgSO}_{4} \cdot 7 \mathrm{H}_{2} \mathrm{O}, 0.4 \mathrm{~g} \mathrm{NaCl}, 0.05 \mathrm{~g} \mathrm{CaCl}_{2} \cdot 2$ $\mathrm{H}_{2} \mathrm{O}, 0.01 \mathrm{~g}$ Fe-Citrate, $1.0 \mathrm{~g}\left(\mathrm{NH}_{4}\right)_{2} \mathrm{SO}_{4}, 1.12 \mathrm{~mL} 10 \mathrm{M}$ $\mathrm{NaOH}$, and $1 \mathrm{~mL}$ trace metals per liter. The tracemetal solution consisted of $0.25 \mathrm{~mL}$ concentrated $\mathrm{HCl}$, $70.0 \mathrm{mg} \mathrm{ZnCl}$, $100.0 \mathrm{mg} \mathrm{MnCl} \cdot 4 \mathrm{H}_{2} \mathrm{O}, 60.0 \mathrm{mg} \mathrm{H}_{3} \mathrm{BO}_{3}$, $200.0 \mathrm{mg} \mathrm{CoCl} \cdot 6 \mathrm{H}_{2} \mathrm{O}, 20.0 \mathrm{mg} \mathrm{CuCl}{ }_{2} \cdot 2 \mathrm{H}_{2} \mathrm{O}, 20.0 \mathrm{mg}$ $\mathrm{NiCl}_{2} \cdot 6 \mathrm{H}_{2} \mathrm{O}$, and $40.0 \mathrm{mg} \mathrm{Na} \mathrm{MoO}_{4} \cdot 2 \mathrm{H}_{2} \mathrm{O} \mathrm{L}{ }^{-1}$. After autoclaving, $1.0 \mathrm{~g}$ sterile butyric acid was added as the substrate. No toxicity or inhibition of $R$. palustris growth was detected at this concentration (Additional file 1). $1 \mathrm{~mL}$ trace vitamins was added to the medium. The trace-vitamin solution consisted of $1.0 \mathrm{~g} p$-aminobenzoic acid, $1.0 \mathrm{~g}$ thiamine, and $0.1 \mathrm{~g}$ biotin $\mathrm{L}^{-1}$. Sterile medium $(50 \mathrm{~mL}$ ) with a final $\mathrm{pH}$ of 7.0 was added to autoclaved serum bottles and sparged for $20 \mathrm{~min}$ with sterile-filtered 80:20 $\mathrm{N}_{2} / \mathrm{CO}_{2}$ gas. For the growth of engineered strains under restrictive butyrate-to-butanol conversion conditions, $\mathrm{NaHCO}_{3}$ was omitted from the medium, the addition of $10 \mathrm{M} \mathrm{NaOH}$ was increased to $2.3 \mathrm{~mL}$ total, and serum bottles were sparged for 20 min with $\mathrm{N}_{2}$ instead of $\mathrm{N}_{2} / \mathrm{CO}_{2}$. In addition, $200 \mu \mathrm{g} \mathrm{mL}{ }^{-1}$ of kanamycin sulfate was added for retention of the plasmid in all growth conditions with engineered strains. All serum bottle cultures were grown in an environmental growth chamber (GC82VH, EGC, Chagrin Falls, OH) at $30{ }^{\circ} \mathrm{C}$ with $80 \mu \mathrm{mol}$ of photosynthetically active photons $\mathrm{s}^{-1} \mathrm{~m}^{-2}$ (photons between 400 and $700 \mathrm{~nm}$ ) illumination from both fluorescent and incandescent lamps.

\section{DNA manipulation and cloning}

We used the green fluorescence protein GFPmut2 [18] as a reporter protein to screen for the activity of different expression vectors in $R$. palustris under aerobic conditions. We tested two different plasmids as candidate expression vectors: (1) the endogenously derived pMG105 [19]; and (2) the broad-host pBBR1MCS-2 [20]. For detection by immunoreaction, we introduced a C-terminal 6X His tag prior to the termination site with PCR by the reverse primer (Table 1). GFPmut2 was inserted into the pMG105 and pBBR1MCS-2 vectors under the control of a range of promoters,

Table 1 Sequences of primers used for this study

\begin{tabular}{|c|c|c|c|}
\hline Primer & Length & Sequence $\left(5^{\prime} \rightarrow 3^{\prime}\right)$ & Purpose \\
\hline \multicolumn{4}{|l|}{ pBBR1MCS-2 vector } \\
\hline Xhol GFPmut2 F1 & 60 & GGCGGCCTCGAGAGGAGGATCTATTCATGAGTAAAGGAGAAGAACTTTTCACTGGAGTTG & Cloning GFPmut2 \\
\hline Xbal GFPmut2 R1 & 51 & GCCGCCTCTAGACTATTTGTATAGTTCATCCATGCCATGTGTAATCCCAGC & \\
\hline BamHI RBS AdhE2 ${ }_{824} \mathrm{~F} 1$ & 68 & $\begin{array}{l}\text { GCCGCGGATCCAGGAGGATCTATTCATGAAAGTTACAAATCAAAAAGAACTAAAACAAAA- } \\
\text { GCTAAATG }\end{array}$ & Cloning adhE2 ${ }_{824}$ \\
\hline Sacl 6X His AdhE2 824 R1 & 62 & CACCCGGAGCTCTAAGTGGTGATGGTGATGATGAAATGATTTTATATAGATATCCTTAAGTT & \\
\hline Sacl AdhE2 ${ }_{824}$ R1 & 43 & CACCCGGAGCTCTAAAATGATTTTATATAGATATCCTTAAGTT & \\
\hline Xhol RBS AdhE $E_{\text {BisB18 }} \mathrm{F} 1$ & 57 & GACGACCTCGAGAGGAGGATCTATTCGTGACCTTATCTACCCCGTCCGACCTCGACA & Cloning adh $E_{\text {BisB18 }}$ \\
\hline Xbal 6X His AdhE $E_{B i s B 18}$ R1 & 60 & CACCCGTCTAGACTAGTGGTGATGGTGATGATGTTCCGCGGCGTTCGCCGTCGCCACCGA & \\
\hline Xbal AdhE $\mathrm{BisB18}_{18} \mathrm{R} 1$ & 52 & CAGTCACCCGTCTAGACTATTCCGCGGCGTTCGCCGTCGCCACCGACAATGT & \\
\hline \multicolumn{4}{|l|}{ pMG105P vector } \\
\hline Xbal GFPmut2 F1 & 60 & GGCGGCTCTAGAAGGAGGATCTATTCATGAGTAAAGGAGAAGAACTTTTCACTGGAGTTG & Cloning GFPmut2 \\
\hline Sall GFPmut2 R1 & 53 & GGCGGCCGGTCGACCTATTTGTATAGTTCATCCATGCCATGTGTAATCCCAGC & \\
\hline
\end{tabular}


including the endogenous phosphoenolpyruvate carboxykinase (ppckA) from $R$. palustris, arabinose (ara), and lactose (lac) promoters. The $a d h E_{\mathrm{Bis} B 18}$ and $a d h E 2_{824}$ genes were amplified from $R$. palustris strain BisB18 and C. acetobutylicum ATCC 824, respectively, using Phusion HF polymerase (New England Biolabs, Ipswich, MA) and directionally ligated into the pBBR1MCS-2 expression vector using the $\mathrm{XhoI} / \mathrm{XbaI}$ cloning sites. Restriction and ligation enzymes were purchased from Promega (Fitchburg, WI) and all constructs were verified with Sanger sequencing (Cornell University Genomics Facility, Ithaca, $\mathrm{NY}$ ). Escherichia coli DH5 $\alpha$ was used for routine cloning of constructs. Both E. coli and R. palustris were transformed by electroporation with a Bio-Rad Gene Pulser II Electroporator (Bio-Rad, Hercules, CA) with $1.8 \mathrm{kV}$, $200 \Omega, 25 \mu \mathrm{F}$ for $E$. coli, and $2.5 \mathrm{kV}, 400 \Omega, 25 \mu \mathrm{F}$ for $R$. palustris. Electroporated cells were recovered in SOC for 1 and $3 \mathrm{~h}$ before plating on lysogeny broth (LB) agar plates with 50 and $200 \mu \mathrm{g} \mathrm{mL}^{-1}$ kanamycin sulfate for isolation of individual $E$. coli and $R$. palustris transformants, respectively.

To successfully produce AdhE2 $2_{824}$ in R. palustris, codon optimization was pursued. Optimization design was performed in-house and largely utilized the one amino acid one codon approach, except in cases of multiple codon repeats and unfavorable energetics of mRNA secondary structure (Additional file 2). adhE2 $2_{\text {opti }}$ with a C-terminal 6X His tag was synthesized by GenScript (Piscataway, $\mathrm{NJ})$ with flanking $\mathrm{XhoI} / \mathrm{XbaI}$ restriction sites that were used for directional cloning into pBBR1MCS-2.

\section{SDS-PAGE and western blot detection}

For both SDS-PAGE and subsequent western blot detection, $50 \mathrm{~mL}$ of late-log E. coli and R. palustris cells were harvested by centrifugation $\left(10 \mathrm{~min}, 12,000 \times g, 4{ }^{\circ} \mathrm{C}\right)$ and resuspended in $1 \mathrm{~mL} 1 \mathrm{X}$ Laemmli sample buffer [21] with a protease inhibitor (Protease inhibitor cocktail, Promega, Fitchburg, WI). The suspension was lysed by ultrasonication on ice using three $30 \mathrm{~s}$ on $1 \mathrm{~min}$ off pulses of $20 \mathrm{~W}$ (Branson Sonifier 150, Emerson, Danbury, CT). After sonication, debris was removed by centrifugation and the total soluble protein concentration was determined by BCA assay (Pierce BCA protein assay kit, Thermo Scientific, Rockford, IL). Forty-five $\mu \mathrm{L}$ of $3 \mathrm{mg} \mathrm{mL}^{-1}$ soluble protein samples and $10 \mu \mathrm{L}$ of Precision Plus Protein ${ }^{\mathrm{TM}}$ standard (Bio-Rad, Hercules, CA) were loaded into pockets of a Mini-PROTEAN ${ }^{\circledR}$ TGX gel (Bio-Rad, Hercules, CA) and run at $100 \mathrm{~V}$ for $1.5 \mathrm{~h}$ before blotting onto PVDF membranes at $100 \mathrm{~V}$ for $1 \mathrm{~h}$. For the initial western blot, immunoreactive bands were developed using manufacturer-suggested protocols for the $1 \mathrm{~h}$ Western $^{\mathrm{TM}}$ Standard Kit for rabbit (GenScript, Piscataway, NJ) and a 6X His primary antibody (Rabbit polyclonal, GeneTex, Irvine, CA). We used a similar protocol a second time to improve the sensitivity with these changes: we used $0.1 \mathrm{~mL} \mathrm{OD}{ }_{600} 1.0$ cells with $0.1 \mathrm{~mL}$ Laemmli sample buffer. The samples were boiled for $10 \mathrm{~min}$ before centrifugation. $15 \mu \mathrm{L}$ of each sample was loaded into separate gel lanes. For development, an enhanced chemiluminescence protocol was used using the manufacturer-suggested protocol for promega ECL western blotting substrate (Fitchburg, WI). The film was allowed to incubate with the PVDF membrane for $30 \mathrm{~min}$ prior to development.

\section{Bioreactor configuration and operation}

Prior to operation, sealed glass bioreactors were autoclaved and filled with $350 \mathrm{~mL}$ sterile RM with $1 \mathrm{~g} \mathrm{~L}^{-1}$ butyrate and without $\mathrm{HCO}_{3}{ }^{-}$. The bioreactors were equipped with a recirculating water jacket that was operated at $30^{\circ} \mathrm{C}$. Magnetic stir plates (IKA Works Inc., Wilmington, NC) provided stirring of the reactors at $450 \mathrm{rpm}$. The bioreactors were continuously sparged with sterile-filtered $\mathrm{N}_{2}$ gas at a flow rate of $0.1 \mathrm{~L} \mathrm{~min}^{-1}$ to maintain an anaerobic environment. After sparging for $1 \mathrm{~h}$ to ensure anaerobic reactor conditions, $1 \mathrm{~mL}$ of actively growing $R$. palustris cells at an $\mathrm{OD}_{600}$ of 0.1 was inoculated into the bioreactor. The preculture of $R$. palustris pBBR1MCS-2 lacp adhE2 $2_{\text {opti }}$ was grown in anaerobic RM without $\mathrm{HCO}_{3}{ }^{-}$, while the preculture of $R$. palustris pBBR1MCS-2 lacp GFPmut2 (control) was grown in aerobic RM without $\mathrm{HCO}_{3}{ }^{-}$. Gas entered the liquid phase of each bioreactor through a sparging stone to increase the gas/liquid interface for butanol stripping. Off-gas was connected to 2 Friedrich's condensers in parallel with recirculating water at $5{ }^{\circ} \mathrm{C}$ to condense the butanol that had been removed by gas stripping. Gas was released into the atmosphere after one passing through the condensers. The bioreactor volume was illuminated externally with a single $60-\mathrm{W}$ incandescent lamp as used previously [22]. The bioreactors were operated in batch-mode and liquid samples were periodically taken for $\mathrm{OD}_{600}$ and metabolite measurements.

\section{Metabolite detection}

Butyrate, butanol, and acetate were measured via HPLC (600 HPLC, Waters, Milford, MA) with a refractive index detector and an Aminex HPX-87H column (BioRad, Hercules, CA). The column temperature was set to $60{ }^{\circ} \mathrm{C}$, and a $5-\mathrm{mM}$ sulfuric acid eluent at a flow rate of $0.6 \mathrm{~mL} \mathrm{~min}^{-1}$ was used as the mobile phase. Butanol and butyraldehyde were measured with a gas chromatograph (HP 5890, Hewlett Packard, Palo Alto, CA) with a 7673 autoinjector and flame ionization detector. The gas chromatograph contained a custom-made packed bed glass column of $1.8 \mathrm{~m} \times 2 \mathrm{~mm}$ id (Supelco, Sigma-Aldrich, 
St. Louis, MO). The support matrix of the column was Chromosorb W/AW80 over 100 mesh; phases were preconditioned: phase A was 10\% Carbowax-20M; phase B was $0.1 \%$ phosphoric acid. The inlet and detector temperatures were 220 and $240{ }^{\circ} \mathrm{C}$, respectively. The column temperature profile was $100{ }^{\circ} \mathrm{C}$ for $2 \mathrm{~min}$, a ramp of $40^{\circ} \mathrm{C} \mathrm{min}^{-1}$ to $180^{\circ} \mathrm{C}$ with a 5 -min hold. Butanol concentrations were verified by both HPLC and GC platforms.

\section{Results and discussion}

\section{GFP screen of expression vectors in R. palustris CGA009}

Different combinations of promoter and vector sequences were screened for highest levels of expression within $R$. palustris. The endogenous ppckA promoter of $R$. palustris is one of the few promoter sequences that had been previously validated to be active in $R$. palustris under gluconeogenic conditions [23]. However, we only observed a minimal increase in fluorescence for both the pMG105 and pBBR1MCS-2 ppckAp GFPmut 2 constructs compared to the empty vector controls (Fig. 2). While the pBBR1MCS-2 arap GFPmut2 construct yielded slightly higher levels compared to $p p c k A p$, the pBBR1MCS-2 lacp GFPmut2 construct offered the best expression overall and was selected as the vector for all further expressions in R. palustris (Fig. 2). Expression from the lac promoter in $R$. palustris was not affected by the addition of the inducer IPTG and catabolite repression only showed a minor decrease in fluorescence (Additional file 3). This suggests that the lac regulatory response system utilized by $E$. coli is not functional in $R$. palustris. Although the pBBR1MCS-2 lac promoter system provided the best expression in R. palustris of all the combinations tested (Fig. 2), the fluorescent signal was still $\sim 13$ times lower than the signal from E. coli pBBR1MCS lacp GFPmut2 (data not shown).

\section{Engineering $a d h E_{\mathrm{BisB18}}$ in R. palustris}

As determined by the lack of growth under restrictive conditions, the absence of butanol production (data not shown), and the lack of a signal in a western blot under permissive conditions (Fig. 3a, AdhE $E_{\text {BisB18 }}$ Aerobic), direct introduction of $a d h E 2_{824}$ in the pBBR1MCS-2 lacp construct did not result in the production of AdhE2 $2_{824}$ in R. palustris. This is likely due to the large GC discrepancy (30\% vs. 65\%) and codon utilization preference between C. acetobutylicum and R. palustris [24]. To address this, we looked for homologous proteins within $R$. palustris. Indeed, endogenous alcohol/aldehyde dehydrogenases with homology to AdhE2 $2_{824}$ have been found to function in the production of butanol from butyrate when overexpressed. This was previously demonstrated with the fucO gene in E. coli, where overexpression allowed production of butanol from an engineered reverse $\beta$-oxidation pathway where no butanol production was observed

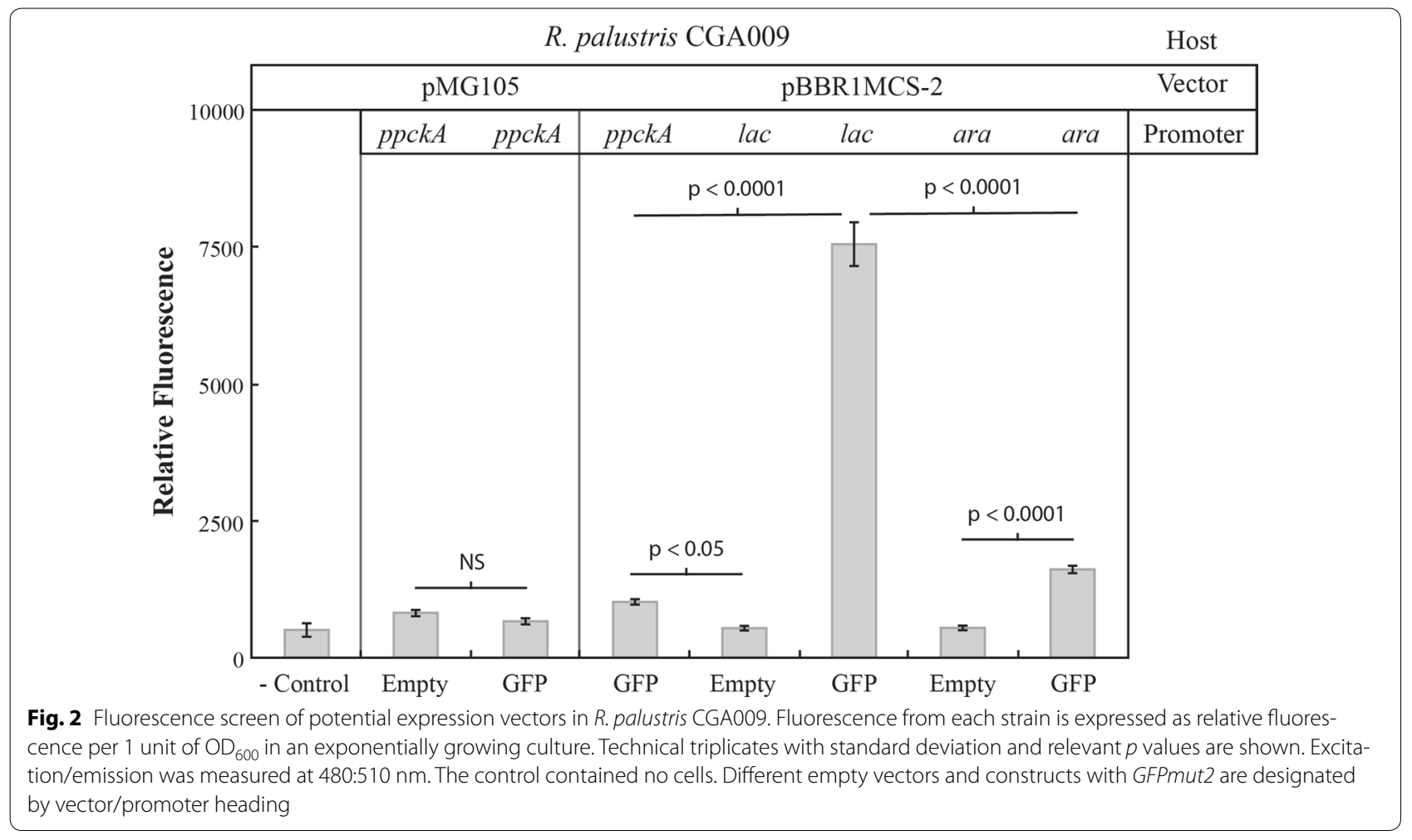




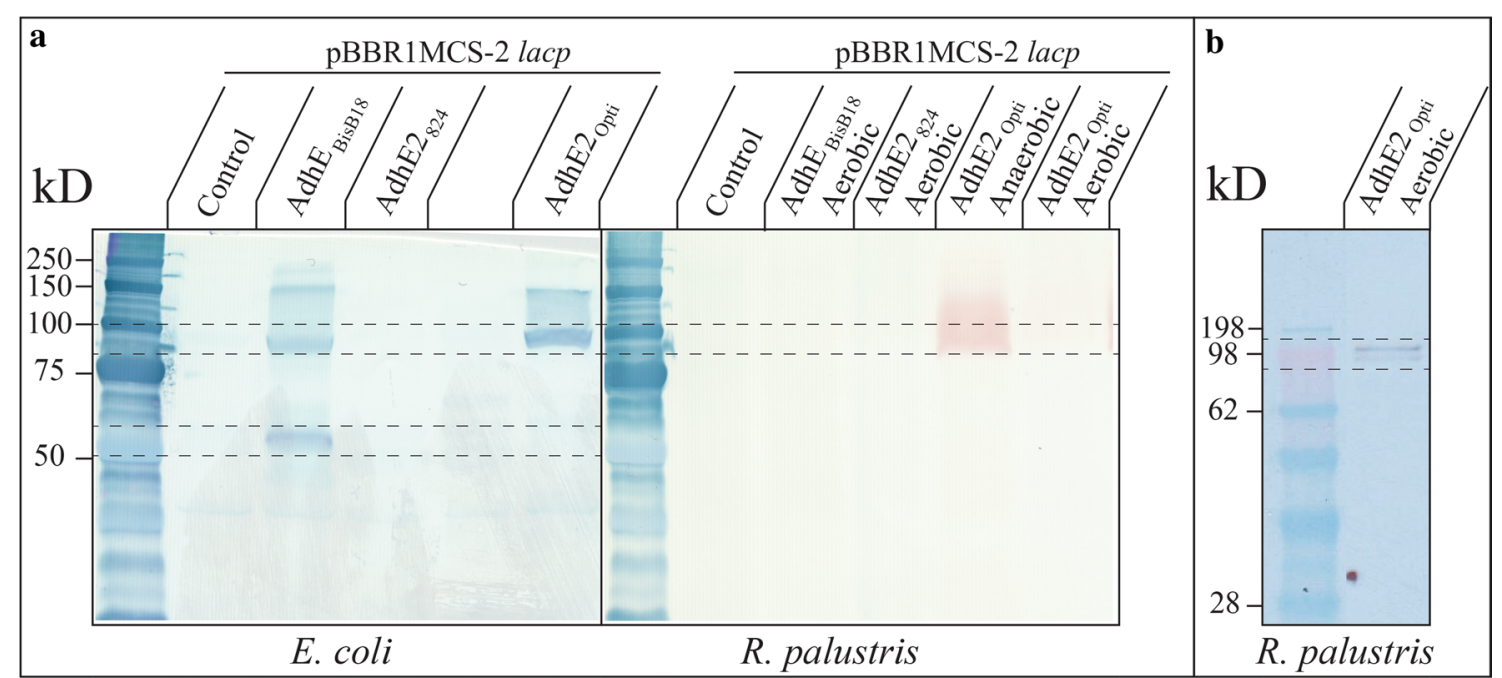

Fig. 3 Western blots for the detection of AdhE2 via the C-terminal 6 X His tag: a in both E. coli and R. palustris. Expected AdhE $E_{B i s B 18,}$ AdhE22 ${ }_{824}$ and AdhE2 ${ }_{\text {opti }}$ sizes are $94.7,94.3$, and $94.3 \mathrm{kD}$, respectively (outlined with dotted box). Downfield band of $\sim 50 \mathrm{kD}$ (outlined with dotted box) likely corresponds to the fragmented C-terminal alcohol dehydrogenase domain where the 6X His tag is located. Protein amounts of $135 \mu \mathrm{g}$ were loaded into all sample lanes. The red band in the R. palustris anaerobic lane is from photo pigments and may partially be obscuring signal from the western blot: b in $R$. palustris pBBR1MCS-2 lacp adhE2 $2_{\text {opti }}$ Cultures. The signal size of aerobic cultures of $R$. palustris pBBR1MCS-2 lacp adhE2 opti Corresponded with the expected size of $A d h E 2_{\text {opti }}(94.3 \mathrm{kD})$

previously [25]. In an attempt to enable butanol production in $R$. palustris, a BLAST search for an endogenous enzyme yielded the identification of RPC_4481 $\left(\operatorname{AdhE}_{\text {BisB18}}\right)$ in R. palustris strain BisB18, which is an aldehyde/alcohol dehydrogenase with high homology to both domains of AdhE2 2824 .

Before expressing $a d h E_{\mathrm{BisB} 18}$ as a candidate butanolpathway gene, $R$. palustris strain BisB18 was screened for its ability to grow by producing butanol from butyrate under restrictive electron sink conditions (without $\mathrm{HCO}_{3}{ }^{-}$). Although no growth or butanol production occurred (data not shown), it is possible that wild-type production of this protein under these conditions is either inactive or insufficient for an observable effect, similar to the FucO protein in E. coli [25]. Because no experimental studies have previously characterized the activity of $\mathrm{AdhE}_{\mathrm{BisB} 18}$, its highly conserved primary sequence was further investigated for its structural homology to the verified butanol-producing AdhE2 $2_{824}$.

Using Swiss-Pdbviewer/DeepView [26], three-dimensional structures of the individual protein domains for both AdhE2 $2_{824}$ and $A d h E_{\text {BisB18 }}$ were generated. The predicted tertiary structure of both aldehyde/alcohol dehydrogenases appeared highly conserved with regard to overall shape, location, active site residues, channel volume, and surface charge (Additional file 4). This suggested that the two proteins likely recognized similar substrates. We, therefore, expressed $a d h E_{\mathrm{BisB} 18}$ in $R$. palustris using the pBBR1MCS-2 lacp construct. Transformants were inoculated into RM with butyrate and with and without $\mathrm{HCO}_{3}{ }^{-}$to screen for viability and butanol production, respectively. Although transformants grew up with $\mathrm{HCO}_{3}{ }^{-}$, no growth was observed without $\mathrm{HCO}_{3}{ }^{-}$condition (data not shown), suggesting that either $\mathrm{Adh}_{\mathrm{BisB} 18}$ was not successfully produced by R. palustris CGA009 or did not have butyryl-CoA-tobutanol conversion activity.

To investigate this further, a crude cell extract of the culture with $\mathrm{HCO}_{3}{ }^{-}$, along with E. coli harboring the same construct, was analyzed via western blotting. Although the strong signal at the expected size of $94.5 \mathrm{kD}$ for $\mathrm{AdhE}_{\mathrm{BisB} 18}$ for $E$. coli $\mathrm{pBBR} 1 \mathrm{MCS}-2$ lacp $a d h E_{\mathrm{BisB} 18}$ demonstrated that the protein was produced (Fig. 3a, $\mathrm{Adh}_{\mathrm{BisB18}}$ ), the lack of a signal from $R$. palustris $\mathrm{pBBR} 1 \mathrm{MCS}-2$ lacp adh $E_{\mathrm{BisB} 18}$ suggested that the protein was either not produced or was produced at levels below detection (Fig. 3a, AdhE $\mathrm{BisB}_{18}$ Aerobic). Aerobic conditions were preferred since anaerobic cultures often had photosynthetic pigments overlapping the expected size for AdhE (Fig. 3a, AdhE $2_{\text {opti }}$ Anaerobic). The lack of growth and butanol production under restrictive conditions, however, demonstrated conclusively that $R$. palustris pBBR1MCS-2 lacp adh $E_{\text {BisB18 }}$ was not endowed with butyrate-to-butanol conversion functionality (data not shown).

\section{Engineering adhE2 opti $_{\text {in }}$ R. palustris}

Another route to circumvent the large GC discrepancy and codon utilization preference between $C$. acetobutylicum and R. palustris was to perform codon optimization 
on $a d h E 2_{824}$ to enable expression. The codon utilization table for $R$. palustris CGA009 was determined by analysis of all genomic coding sequences and was retrieved from the Kazusa database [27]. The $a d h E 2_{\text {opti }}$ gene resulted in an increase of the average codon utilization frequency in R. palustris from $23 \%$ for the original adhE2 $2_{824}$ to $64 \%$ for the optimized $a d h E 2_{\text {opti }}$ (Additional file 5). Codon optimization resulted in a shift of the GC content from $32.5 \%$ for $a d h E 2_{824}$ to $62 \%$ for $a d h E 2_{\text {opti }}$. In addition, the presence of rare codons $(<10 \%$ utilization frequency), which previously composed $47.4 \%$ of all codons in $a d h E 2_{824}$, was entirely eliminated. One disadvantage to this approach of codon optimization is that rare codons are known to induce ribosomal pausing, which has been demonstrated important for proper folding of proteins. Thus, with the elimination of all rare codons from $a d h E 2_{\text {opti, }}$, the elimination of codon-dependent ribosomal pausing could lead to improper folding and aggregation of the target protein [28]. For comparison with $a d h E 2_{\text {opti, }}$, the codon utilization frequency of $a d h E_{\mathrm{BisB} 18}$ averaged 55\% (Additional file 5), the gene possessed an overall $63 \%$ GC content, and the frequency of rare codons within the gene was $5 \%$, demonstrating the utilization of rare codons for proper translation of proteins in endogenous alcohol/aldehyde genes (Additional file 6).

To test whether $a d h E 2_{\text {opti }}$ would be expressed in $R$. palustris CGA009 and enable growth by conversion of butyrate to butanol, the gene was introduced using the pBBR1MCS-2 lacp construct. Transformants were then inoculated into RM with butyrate and with and without $\mathrm{HCO}_{3}{ }^{-}$for growth screening and western blot analysis. This engineered strain was able to grow without $\mathrm{HCO}_{3}{ }^{-}$(restrictive conditions) and it produced a measureable amount of butanol, demonstrating the successful production of AdhE2 $2_{\text {opti }}$ with a functional activity. While a detectable signal was observed on a western blot for the E. coli cloning culture, no signal was initially detected from $R$. palustris pBBR1MCS-2 lacp adhE2 $2_{\mathrm{opti}}$ (Fig. 3a, AdhE2 $2_{\text {opti }}$ Anaerobic and AdhE2 $2_{\text {opti }}$ Aerobic). We performed a second western blot using a more sensitive X-ray film imaging method. For aerobically grown R. palustris pBBR1MCS-2 lacp adhE2 $2_{\mathrm{opti}}$, we observed a single signal at approximately $94 \mathrm{kD}$, demonstrating successful expression (Fig. 3b, AdhE2 $2_{\text {opti }}$ Aerobic). As stated above, GFPmut2 production from the pBBR1MCS lac promoter is much lower in R. palustris than in E. coli, so the lack of a signal in the majority of the samples is likely due to low protein production in $R$. palustris.

\section{Butanol production rates and selectivity for $R$. palustris pBBR1MCS-2 lacp adhE2 ${ }_{\text {opti }}$}

Because of the rescued growth and butanol production observed, $R$. palustris pBBR1MCS-2 lacp adhE2 ${ }_{\text {opti }}$ was further investigated for its ability to grow under both permissive and restrictive conditions and these results were compared to R. palustris pBBR1MCS-2 lacp GFPmut2 as a control. $R$. palustris pBBR1MCS-2 lacp adhE2 ${ }_{\text {opti }}$ was capable of growth without $\mathrm{HCO}_{3}{ }^{-}$(restrictive conditions) (Fig. 4b), while this growth did not occur for the control strain. The growth for $R$. palustris pBBR1MCS-2 lacp adhE2 $2_{\text {opti }}$ occurred on a long time scale and reached a relatively low maximum cell density of $\sim 0.06 \mathrm{OD}_{600}$ (Fig. 4b) at neutral $\mathrm{pH}$ levels (Fig. 4d). Following the onset of the stationary phase, consumption of butyrate and production of butanol still occurred (Fig. 4f, h), but the cultures only reached a maximum butanol concentration of $<0.4 \mathrm{mM}$ after extended incubation (Fig. 4h).

Although only low levels of butyrate were consumed (Fig. 4f), butanol production occurred with a $39.7 \pm 7.6 \%$ selectivity based on moles of carbon. The theoretical maximum for butanol production is based on all electrons derived from complete oxidation of one molecule of butyrate reducing five molecules of butyrate to butanol ( 6 butyrate $\rightarrow 5$ butanol $+4 \mathrm{CO}_{2}$ ) for $83 \%$ conversion efficiency. However, based on previous empirical measurements of excess reducing equivalents of $R$. palustris growing with butyrate by McKinlay et al. [3], only an estimated $0.45 \mathrm{~mol}$ of butanol should be produced per mole of butyrate consumed for an effective $45 \%$ selectivity ( 6 butyrate $\rightarrow 2.7$ butanol + biomass). Therefore, almost all reducing equivalents predicted to be in excess were being converted into butanol by $R$. palustris pBBR1MCS-2 lacp $a d h E 2_{\text {opti }}(40 \%$ vs. $45 \%)$, which we had anticipated for an obligatory reaction.

To further investigate why $R$. palustris pBBR1MCS-2 lacp adhE2 $2_{\text {opti }}$ showed low growth rates, $R$. palustris was screened for product (butanol) toxicity. However, no inhibition of growth was observed in $R$. palustris at butanol concentrations up to $\sim 30 \mathrm{mM}$ (Additional file 7), removing our concerns about a concentration of $\sim 0.4 \mathrm{mM}$ (Fig. 4h). In addition, we operated a bioreactor with product removal to investigate whether this would increase growth and butanol production rates by removing butanol from the substrate pool, thereby, improving the thermodynamics for butanol formation and eliminating any end-product inhibition. Sparging of $\mathrm{N}_{2}$ has been previously used to remove butanol from fermentation broths [29]. Here, $\mathrm{N}_{2}$ gas was continuously sparged into $350-\mathrm{mL}$ anaerobic bioreactors to remove butanol produced by $R$. palustris pBBR1MCS-2 lacp adhE2 $2_{\text {opti }}$ in batch-mode for 6 consecutive weeks. The R. palustris pBBR1MCS-2 lacp adhE2 $2_{\text {opti }}$ culture grew for that entire period up to an $\mathrm{OD}_{600}$ of 0.12 (Additional file 8: Fig. S6A), and achieved an average maximum butanol production rate of $0.0017 \mathrm{~g} \mathrm{~L}^{-1}$ day $^{-1}$ (Additional file 8: Fig. S6D) with a maximum butanol concentration of 

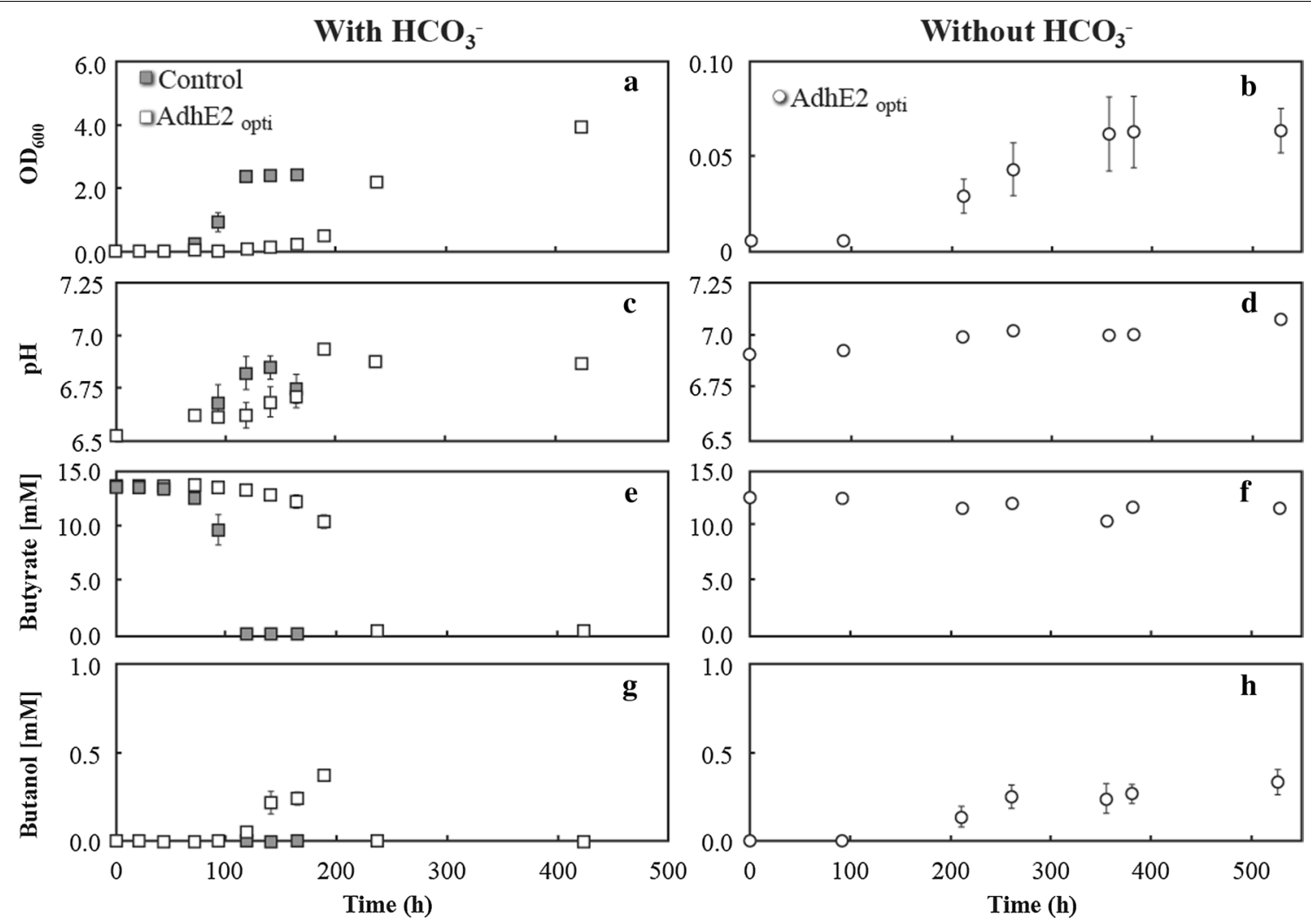

Fig. 4 Performance of R. palustris pBBR1MCS-2 lacp GFPmut2 (control) and R. palustris pBBR1MCS-2 lacp adhE2 opti in permissive conditions (with $\mathrm{HCO}_{3}{ }^{-}$, squares), and R. palustris pBBR1MCS-2 lacp adhE2 ${ }_{\text {opti }}$ in restrictive conditions (without $\mathrm{HCO}_{3}{ }^{-}$, circles): $\mathbf{a}, \mathbf{b}$ growth; $\mathbf{c}, \mathbf{d} \mathrm{pH} ; \mathbf{e}, \mathbf{f} \mathrm{concentra-}$ tion of butyrate in the broth; $\mathbf{g}, \mathbf{h}$ butanol concentration in the broth. The results from the control and R. palustris pBBR1MCS-2 lacp adhE2 opti $\operatorname{are}$ represented by gray and white symbols, respectively. Average and standard deviation of biological triplicates are shown

$0.35 \mathrm{mM}$ (Additional file 8: Fig. S6C) without depleting butyrate (Additional file 8: Fig. S6B). The lack of a considerable improvement compared to the earlier experiment showed that neither butanol toxicity nor thermodynamics nor end-product inhibition was a main reason for low growth rates. Finally, we found that the extraction system removed a majority of the butanol without removing any of the butyrate (Additional file 8: Fig. S6E, F).

The intermediate butyraldehyde within microbial cells is another metabolite that could have inhibited $R$. palustris, even though the maximum concentration in the supernatant was measured to be only $50 \mu \mathrm{M}$. However, due to the fusion nature of AdhE $2_{\text {opti, }}$, we anticipate that butyraldehyde would rapidly be converted into butanol with a high flux rate, resulting in a low effective concentration [30]. Without butanol and butyraldehyde toxicity, we can only speculate about why growth and butanol production rates of our engineered strain are so low. Since AdhE2 $2_{\text {opti }}$ is provided as the sole route to maintaining redox balance, all excess electrons must be funneled through this pathway. When the capacity of the AdhE2 $2_{\text {opti }}$ pool is outweighed by the demand for regenerating oxidized reducing equivalents, this would introduce a bottleneck in the maximum rate of metabolism and growth. However, more work is required to be conclusive.

Some butanol production did also occur for R. palustris pBBR1MCS-2 lacp adhE2 ${ }_{\text {opti }}$ with $\mathrm{HCO}_{3}{ }^{-}$(permissive conditions) when exogenous $\mathrm{CO}_{2}$ was available for maintaining redox balance (Fig. 4a, g) at a neutral $\mathrm{pH}$ level (Fig. 4c). Because the preculture had originally been maintained without $\mathrm{HCO}_{3}^{-}$, a long lag phase and carryover of butanol producing activity was observed for $R$. palustris pBBR1MCS-2 lacp adhE2 $2_{\text {opti }}$ (Fig. 4a), resulting in a maximum concentration of $0.37 \mathrm{mM}$ with $11.5 \pm 2.4 \%$ selectivity (Fig. $4 \mathrm{e}, \mathrm{g}$ ). Though the transient production of butanol was a surprise, this lower selectivity for the permissive condition was expected because more reducing equivalents could be used in central metabolism following the introduction of exogenous $\mathrm{HCO}_{3}{ }^{-}$, resulting in much higher growth $(\sim 10 \times \mathrm{OD}$ at $200 \mathrm{~h}$ ), the complete depletion of butyrate (Fig. 4e), no production of acetate, and ultimate consumption of all butanol produced (Fig. 4g). This contrasts with the 
restrictive condition where all growth proceeds through the obligate engineered pathway, butyrate is not depleted, and all butanol produced remains in solution.

\section{Optimizing butanol production by pregrowing $R$. palustris pBBR1MCS-2 lacp adhE2 opti}

In an attempt to improve the maximum butanol concentrations produced by the engineered strain, $50 \mathrm{~mL}$ aerobic cultures was grown to mid-log phase, washed 3 times with sterile RM, resuspended in $0.5 \mathrm{~mL} \mathrm{RM}$, and used as an inoculum into $20-\mathrm{mL}$ anaerobic cultures under restrictive conditions. This procedure ensured that the butyrate-to-butanol conversion was not catalyst limited as the initial $\mathrm{OD}_{600}$ of these cultures was $>1$. By using a concentrated inoculum, $R$. palustris pBBR1MCS-2 lacp $\operatorname{adh} E 2_{\text {opti }}$ produced butanol concentrations of greater than $1.5 \mathrm{mM}$ at volumetric butanol production rates of $0.034 \mathrm{~g} \mathrm{~L}^{-1}$ day $^{-1}$ (Fig. 5d, f). All butanol was produced within $100 \mathrm{~h}$ of inoculation (Fig. 5d, f). Following $100 \mathrm{~h}$, however, the $R$. palustris pBBR1MCS-2 lacp adhE2 $2_{\text {opti }}$ culture continued to grow and consume butyrate (Fig. 5a, c) with the main carbon product switching from butanol to acetate (Fig. 5b). Acetate had not been detected before in any of the previous anaerobic experiments and in terms of maintaining redox balance, acetate is an unfavorable redox product compared to butanol. This metabolism reduced the butanol selectivity (Fig. 5e). We do not know exactly why this switch occurred, but it was likely a result from having a high biomass culture that underwent a rapid switch from aerobic to anaerobic conditions.

\section{Conclusion}

The introduction of $a d h E 2_{\text {opti }}$ into $R$. palustris via a plasmid-based construct is an example of metabolically engineered fuel production that proceeds as an obligate strategy for growth. Although growth and production rates were low for this system, our selectivity of $39.7 \pm 7.6 \%$ matched well with the empirical expectation of $45.0 \%$ under these conditions. Because the low growth rates and butanol production are likely constrained by
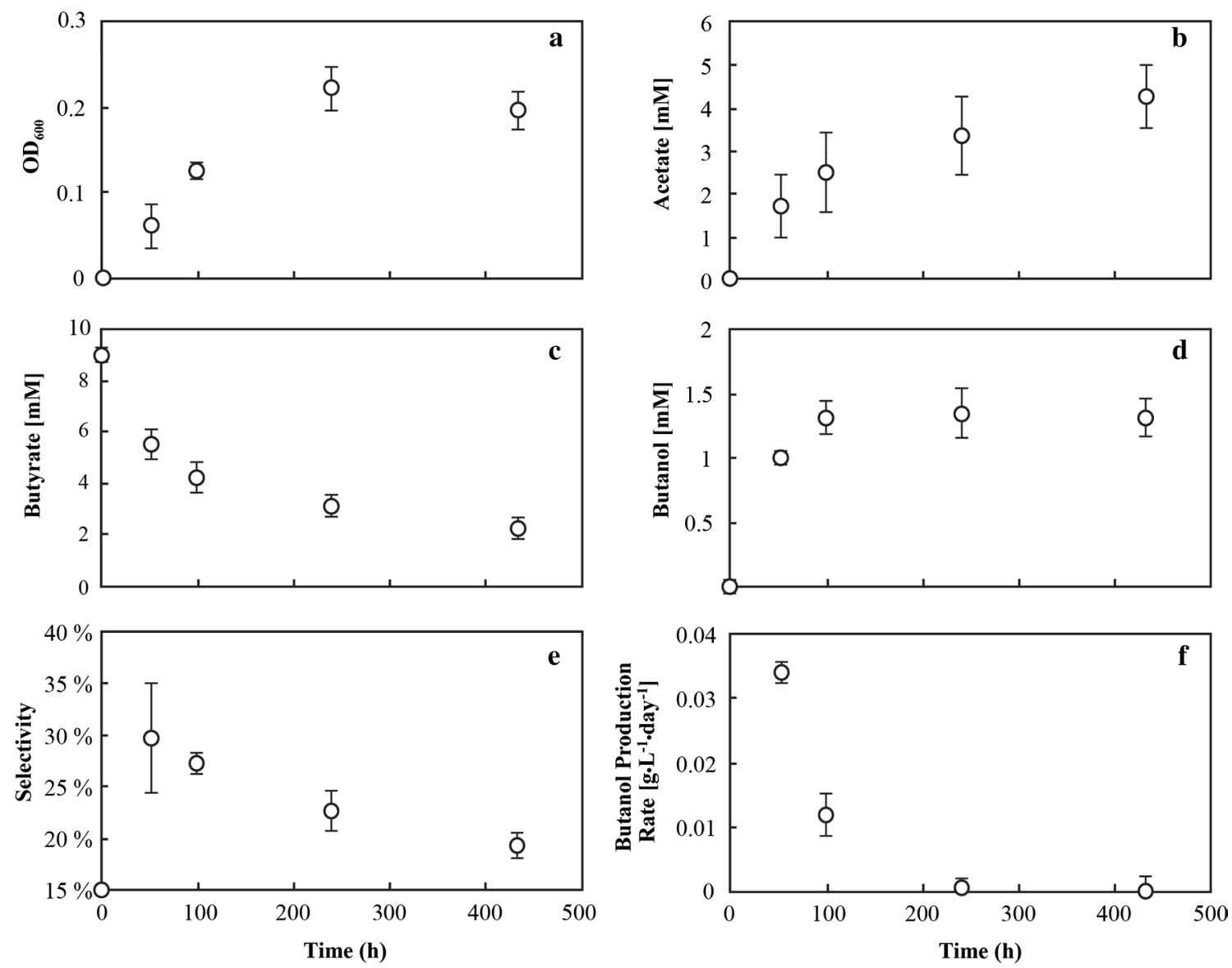

Fig. 5 Performance of R. palustris pBBR1MCS-2 lacp adhE2 ${ }_{\text {opti }}$ without $\mathrm{HCO}_{3}{ }^{-}$in bottles that were inoculated with concentrated cultures: a growth with corrected $\mathrm{OD}_{600}$ (initial $\mathrm{OD}_{600}$ was subtracted); $\mathbf{b}$ acetate concentration in the broth; $\mathbf{c}$ butyrate concentration in the broth; $\mathbf{d}$ butanol concentration in the broth; $\mathbf{e}$ butanol selectivity; $\mathbf{f}$ volumetric butanol production rate. Error bars show the standard error of three biological replicates 
redox flux through the engineered pathway, rational design and directed evolution could be used to further improve the performance of this activity. If improved, the system could be easily integrated into a simple bioreactor design with highly efficient selectivity and recovery. While this proof-of-concept for harnessing an obligate reducing metabolism to drive engineered reactions used butanol as a target molecule, this redox-based driving force could be used to achieve other reactions for the production of desirable chemicals that require reduction reactions.

\section{Additional files}

Additional file 1. Growth with butyrate, containing Figure S1.

Additional file 2. Sequence.

Additional file 3. Fluorescence, containing Figure S2.

Additional file 4. Structure, containing Figure $\mathrm{S3}$.

Additional file 5. Codons, containing Figure $S 4$.

Additional file 6. Codons and GC content, containing Table S1.

Additional file 7. Growth with butanol, containing Figure $\mathrm{S} 5$.

Additional file 8. Growth with product removal, containing Figure S6.

\section{Authors' contributions}

DFRD designed and executed experiments, performed analysis, interpreted data, and drafted the manuscript. ECH executed experiments, performed analysis, and contributed to data interpretation. HR and BM advised on how to perform the experiments. GJ and LTA conceived the study and participated in its design. LTA coordinated the study and contributed to the drafting of the manuscript. All authors read and approved the final manuscript.

\section{Author details}

1 Department of Biological and Environmental Engineering, Cornell University, Ithaca, NY 14853, USA. ${ }^{2}$ Boyce Thompson Institute for Plant Research, Ithaca, NY 14853, USA. ${ }^{3}$ Atkinson Center for a Sustainable Future, Cornell University, Ithaca, NY 14853, USA. ${ }^{4}$ Center for Applied Geosciences, University of Tübingen, Hölderlinstr. 12, 72074 Tübingen, Germany.

\section{Acknowledgements}

We would like to thank Dr. Meena Haribal, Dr. Lubna Richter, Prof. Dr. Matt DeLisa, Dr. Jason Boock, and Jen Schmidt for their expertise and advice (all at Cornell University). We would like to thank Prof. Dr. Caroline Harwood (University of Washington) for providing the R. palustris strain BisB18 and Wendy Hoose (Cornell University) for providing technical assistance.

\section{Competing interests}

The authors declare that they have no competing interests.

\section{Availability of data and materials}

The datasets generated and/or analyzed during the current study are available from the corresponding author on reasonable request.

\section{Funding}

This project and its researchers were funded in part through an Academic Venture Fund award from the Atkinson Center for a Sustainable Future to LTA and GJ, and the Advanced Research Projects Agency Energy Open 2012 program Project Number DE-AR0000312 to LTA. We acknowledge support by Deutsche Forschungsgemeinschaft and Open Access Publishing Fund of University of Tübingen.

\section{Publisher's Note}

Springer Nature remains neutral with regard to jurisdictional claims in published maps and institutional affiliations.

Received: 17 April 2017 Accepted: 28 June 2017

Published online: 11 July 2017

\section{References}

1. McKinlay JB, Harwood CS. Carbon dioxide fixation as a central redox cofactor recycling mechanism in bacteria. Proc Natl Acad Sci USA. 2010;107(26):11669-75.

2. Hillmer $\mathrm{P}$, Gest $\mathrm{H}_{\text {. }} \mathrm{H}_{2}$ metabolism in the photosynthetic bacterium Rhodopseudomonas capsulata: $\mathrm{H}_{2}$ production by growing cultures. J Bacteriol. 1977;129(2):724-31.

3. McKinlay JB, Harwood CS. Calvin cycle flux, pathway constraints, and substrate oxidation state together determine the $\mathrm{H}_{2}$ biofuel yield in photoheterotrophic bacteria. mBio. 2011;2(2):e00323.

4. Barbosa MJ, Rocha JMS, Tramper J, Wijffels RH. Acetate as a carbon source for hydrogen production by photosynthetic bacteria. J Biotechnol. 2001;85(1):25-33

5. Gosse JL, Engel BJ, Rey FE, Harwood CS, Scriven LE, Flickinger MC. Hydrogen production by photoreactive nanoporous latex coatings of nongrowing Rhodopseudomonas palustris CGA009. Biotechnol Prog. 2007;23(1):124-30

6. Chen CY, Saratale GD, Lee CM, Chen PC, Chang JS. Phototrophic hydrogen production in photobioreactors coupled with solar-energy-excited optical fibers. Int J Hydrog Energ. 2008;33(23):6886-95.

7. Fixen KR, Zheng Y, Harris DF, Shaw S, Yang Z-Y, Dean DR, Seefeldt LC, Harwood CS. Light-driven carbon dioxide reduction to methane by nitrogenase in a photosynthetic bacterium. Proc Natl Acad Sci USA. 2016;113(36):10163-7.

8. Jin C, Yao M, Liu H, Lee C-F, Ji J. Progress in the production and application of $n$-butanol as a biofuel. Renew Sustain Energ Rev. 2011;15(8):4080-106.

9. Nolling J, Breton G, Omelchenko MV, Makarova KS, Zeng QD, Gibson R, Lee HM, Dubois J, Qiu DY, Hitti J, et al. Genome sequence and comparative analysis of the solvent-producing bacterium Clostridium acetobutylicum. J Bacteriol. 2001;183(16):4823-38.

10. Inui M, Suda M, Kimura S, Yasuda K, Suzuki H, Toda H, Yamamoto S, Okino S, Suzuki N, Yukawa H. Expression of Clostridium acetobutylicum butanol synthetic genes in Escherichia coli. Appl Microbiol Biotechnol. 2008;77(6):1305-16.

11. Atsumi S, Cann AF, Connor MR, Shen CR, Smith KM, Brynildsen MP, Chou KJ, Hanai T, Liao JC. Metabolic engineering of Escherichia coli for 1-butanol production. Metab Eng. 2008;10(6):305-11.

12. Desai RP, Harris LM, Welker NE, Papoutsakis ET. Metabolic flux analysis elucidates the importance of the acid-formation pathways in regulating solvent production by Clostridium acetobutylicum. Metab Eng. 1999;1(3):206-13.

13. Sillers R, Al-Hinai MA, Papoutsakis ET. Aldehyde-alcohol dehydrogenase and/or thiolase overexpression coupled with CoA transferase downregulation lead to higher alcohol titers and selectivity in Clostridium acetobutylicum fermentations. Biotechnol Bioeng. 2009;102(1):38-49.

14. Dürre P, Fischer RJ, Kuhn A, Lorenz K, Schreiber W, Sturzenhofecker B, Ullmann S, Winzer K, Sauer U. Solventogenic enzymes of Clostridium acetobutylicum: catalytic properties, genetic organization, and transcriptional regulation. FEMS Microbiol Rev. 1995;17(3):251-62.

15. Nair RV, Bennett GN, Papoutsakis ET. Molecular characterization of an aldehyde/alcohol dehydrogenase gene from Clostridium acetobutylicum ATCC 824. J Bacteriol. 1994;176(3):871-85.

16. Fontaine L, Meynial-Salles I, Girbal L, Yang X, Croux C, Soucaille P. Molecular characterization and transcriptional analysis of adhE2, the gene encoding the NADH-dependent aldehyde/alcohol dehydrogenase responsible for butanol production in alcohologenic cultures of Clostridium acetobutylicum ATCC 824. J Bacteriol. 2002;184(3):821-30.

17. Lee $C M$, Chen PC, Wang CC, Tung YC. Photohydrogen production using purple nonsulfur bacteria with hydrogen fermentation reactor effluent. Int J Hydrog Energy. 2002;27(11-12):1309-13. 
18. Cormack BP, Valdivia RH, Falkow S. FACS-optimized mutants of the green fluorescent protein (GFP). Gene. 1996;173(1):33-8.

19. Inui $M$, Roh JH, Zahn $K$, Yukawa H. Sequence analysis of the cryptic plasmid pMG101 from Rhodopseudomonas palustris and construction of stable cloning vectors. Appl Environ Microbiol. 2000;66(1):54-63.

20. Kovach ME, Elzer PH, Hill DS, Robertson GT, Farris MA, Roop RM, Peterson KM. Four new derivatives of the broad-host-range cloning vector pBBR1MCS, carrying different antibiotic-resistance cassettes. Gene. 1995;166(1):175-6.

21. Protocols CSH. Laemmli sample buffer (2X). Cold Spring Harbor Protocols. 2006;2006(1):pdb.rec10424.

22. Doud DFR, Angenent LT. Toward electrosynthesis with uncoupled extracellular electron uptake and metabolic growth: enhancing current uptake with Rhodopseudomonas palustris. Environ Sci Technol Lett. 2014;1 (9):351-5.

23. Inui M, Nakata K, Roh JH, Zahn K, Yukawa H. Molecular and functional characterization of the Rhodopseudomonas palustris no. 7 phosphoenolpyruvate carboxykinase gene. J Bacteriol. 1999;181(9):2689-96.
24. Gustafsson C, Govindarajan S, Minshull J. Codon bias and heterologous protein expression. Trends Biotechnol. 2004;22(7):346-53.

25. Dellomonaco C, Clomburg JM, Miller EN, Gonzalez R. Engineered reversal of the beta-oxidation cycle for the synthesis of fuels and chemicals. Nature. 2011;476(7360):355-9.

26. Guex N, Peitsch MC. SWISS-MODEL and the Swiss-Pdb Viewer: an environment for comparative protein modeling. Electrophoresis. 1997;18(15):2714-23.

27. Nakamura Y, Gojobori T, Ikemura T. Codon usage tabulated from international DNA sequence databases: status for the year 2000. Nucleic Acids Res. 2000;28(1):292.

28. Rosano GL, Ceccarelli EA. Rare codon content affects the solubility of recombinant proteins in a codon bias-adjusted Escherichia coli strain. Microb Cell Fact. 2009;8:41.

29. Qureshi N, Blaschek HP. Recovery of butanol from fermentation broth by gas stripping. Renew Energy. 2001;22(4):557-64.

30. DeLisa MP, Conrado RJ. Synthetic metabolic pipelines. Nat Biotechnol. 2009;27(8):728-9.

\section{Submit your next manuscript to BioMed Central and we will help you at every step:}

- We accept pre-submission inquiries

- Our selector tool helps you to find the most relevant journal

- We provide round the clock customer support

- Convenient online submission

- Thorough peer review

- Inclusion in PubMed and all major indexing services

- Maximum visibility for your research

Submit your manuscript at www.biomedcentral com/submit 